\title{
A reverse logistic inventory model for imperfect production process with preservation technology investment under learning and inflationary environment
}

\author{
Preeti Jawla $^{a^{*}}$ and S. R. Singh ${ }^{\mathrm{b}}$
}

${ }^{a}$ Department of Mathematics, Banasthali University, Rajasthan, 304022, India ${ }^{b}$ Department of Mathematics, D.N. College, Meerut, 250001, India

\begin{tabular}{l}
\hline C H R O N I C L E \\
\hline Article history: \\
Received March 20, 2014 \\
Received in revised format \\
November 18, 2015 \\
Accepted December 182015 \\
Available online \\
December 19 2015 \\
\hline Keywords: \\
Reverse logistics \\
Imperfect production \\
Learning \\
Preservation \\
Multi-items \\
Inflation \\
Exponential holding cost \\
Exponential demand rate
\end{tabular}

\section{A B S T R A C T}

This paper presents a unified multi items general inventory model for integrated production of new items and remanufacturing of returned and defected items for a finite planning horizon. In this paper, a production model that takes into account learning, instantaneous deterioration rate and inflation is proposed. In addition, we also consider that the holding cost is a non-negative, non-decreasing and continuous function of time. In this model, the preservation technology is used to reduce the rate of product deterioration. A theory is developed to find the optimal solution of the proposed model; it is then exemplified with the help of several numerical examples. An efficient solution procedure is also provided to find the optimal strategy. Finally, sensitivity of the optimal solution to changes in the values of different parameters of the system and the convexities of the cost functions are also studied and represented through the graphs.

(C) 2016 Growing Science Ltd. All rights reserved.

\section{Introduction}

Enforced legislation and customer expectations increasingly oblige manufacturers to take back their products after use and remanufacture them. This paper is based the concept of reverse logistics. According to Dekker et al. (2013) Reverse Logistics is: "The process of planning, implementing, and controlling flows of raw materials, in-process inventory, and finished goods, from a manufacturing, distribution or use point to a point of recovery or point of proper disposal". Examples of reverse logistics include recalls, commercial returns, warranties, wrong deliveries, repairs \& refurbishment and end-of-life returns that companies face. Reverse logistics is the process that collects and remanufactures used products to achieve quality standards that are "as-good-as those of new products". Although reverse logistics is relatively a new term, the earliest approach in the area of reverse logistics sizes was made by Schrady (1967). He developed joint determination of production and remanufacturing lot and

* Corresponding author Tel: +91-880-074-4252

E-mail address: p.preeti.jawla@gmail.com (P.Jawla)

C 2016 Growing Science Ltd. All rights reserved. doi: $10.5267 /$ j.uscm.2015.12.001 
analyzed the problem in the traditional Economic Order Quantity (EOQ) model by setting: continuous and deterministic demand and return. Schrady's (1967) model considered by Nahmias and Rivera (1979) for the case of limited storage in the repair and finite repair rate and production shops.

In recent years, the concept of reverse logistics has been developed. Also, many researchers discussed the economic ordering quantity in the general logistics and reverse logistics. Guide et al. (1996) mentioned the characteristic of reverse logistics to analyze the quantity and quality of returned items in reverse logistics. Krumwiede (2002) defined the reverse process of logistics as reverse logistics, and indicated retailers' thought on reverse logistics as the products returned from customers to the sellers. For the manufacturer, the reverse logistics is the process of defective product returning from users. Dobos and Richter (2003) developed a production/recycling model with constant demand that is gratified by recycling with a single repair and a single production cycles and non- instantaneous production per time interval. Singh and Saxena (2012), Yang et al. (2013) and Singh et al. (2013) have investigated their model by using different assumptions and reverse flow of material. There are more and more literatures about reverse logistics, but most of them did not consider the effect of inflation and use of preservation technology.

In the literature of inventory theory, deteriorating inventory models have been continually modified to include more practical characteristics of the real inventory systems. In real life, the deterioration (vaporization, damage, spoilage, dryness and so on) phenomenon is observed on inventory items such as fruits, vegetables, pharmaceuticals, volatile liquids and many others. The analysis of deteriorating inventory model began with Ghare and Schrader (1963), who established an exponentially decaying inventory for a constant demand. However, it has been empirically observed that life expectancy and failure of many items could perform better expressed by a variable deterioration rate. Covert and Philip (1973) extended the model of Ghare and Schrader (1963), for a variable rate of deterioration by assuming Weibull distribution and Gamma deterioration rates, respectively. When determining the optimal inventory policy for that type of products, we cannot ignore the loss due to deterioration.

Ouyang et al. (2006) and $\mathrm{Wu}$ et al. (2006) were the first who incorporated the phenomenon of "noninstantaneous deterioration" into the inventory modeling. In practice, the deterioration rate of manufactured goods can be controlled and reduced through various efforts such as specialized equipment acquisition and procedural changes. It is well known that some products have a high deterioration rate like semiconductor chips, medicine, refrigerated food, and many others. Therefore, many enterprises developed preservation technologies to control causes of deterioration and increase the profit. For agreement with the realistic inventory situation, Wee et al. (2010) presented a deteriorating inventory model with constant demand rate and exponential decay items with timedependent partial backlogging rate. In addition, the retailer is allowed to reduce the rate of product deterioration by investing on the preservation technology. Wee et al. (2010) then extended the model developed by Hsieh and Dye (2012). In this model they assumed that the preservation technology cost was a function of the length of replenishment cycle.

Inflation is a fundamental feature of today's economy all over the world and large-scale inflation rates are not uncommon in many countries. So, while determining the optimal inventory policy, the effects of inflation and time value of money cannot be ignored. Many researchers have developed inventory models with the inflationary effect. Buzacott (1975) was the first who developed the first EOQ model taking effect of inflation into account by considering a constant inflation rate. Several researchers have extended their ideas for different situations considering time value of money and various inflation rates. A multi-item inventory model with a resource constraint system under a variable inflation rate was developed by Gupta and Vrat (1986). Ray and Chaudhuri (1997) investigated an EOQ model under time discounting and inflation allowing shortages. An inventory model with deteriorating items and permissible delay in payments under inflation was analyzed by Liao et al. (2000). Sarkar and Moon (2011) proposed an EPQ model under the effect of inflation with imperfect production. Singh et al. 
(2012) considered a warehouse imperfect fuzzified production model with shortages under inflationary conditions. It is often the case that the performance of a person, group of persons, or an organization, engaged in a repetitive task improves with time. This is due to the learning phenomenon, which is a decrease in the cost and/or the time required to produce each successive unit. For example, the familiarity with operational tasks and their environments, and the effective use of tools and machines are usually increased with repetition. The "Learning Phenomenon" introduced by Wright (Wright, 1936) to study factors affecting the cost of airplanes. The WLC (Wright, 1936) is represented as

$y(x)=y_{1} x^{-b}$,

where $y_{1}$ is the time to produce the first unit, $\mathrm{b}$ is the learning exponent $(0<b<1), x$ the cumulative production, and $y(x)$ is the time to produce (perform) the $x$ th unit (repetition). A comprehensive survey of empirical models for the learning curve was given by Yelle (1979). Several models have been investigated and evolved with the economic order (production) quantity (EOQ/EPQ) model in a reverse logistics and rework context. For example, Garvin (1988) discussed the common sources of improvement for quality and productivity. Lower quality implies rework and higher scrap, which in turn means equipment time, labor, wasted material, and other modalities. Badiru (1995) developed a production model, which includes the costs of producing an item, relearning, and reworks, reduces as production increases according to the power-form learning curve of Wright (1936). An economical evaluation of disassembly operations for remanufacturing, recycling and reuse was considered by Johnson and Wang (1998). Jaber and Bonney (2003) empirically showed that the time required to rework a defective item reduces as production increases and that rework times conform to the learning relationship. Jaber and Saadany (2011) developed an economic production and remanufacturing model with learning effects. Singh et al. (2013) proposed an imperfect production inventory model with inflation and learning under two limited storage capacity.

In the present paper, an attempt has been made to develop a multi-item reverse logistics model with learning effect. An economic production inventory model for integrated production of new items and remanufacturing of returned items is presented for a finite planning horizon and having time dependent demand. Demand is increasing with time. The production, remanufacturing and return, rates are arbitrary functions of time. We have taken the holding cost as a continuous, non-negative and nondecreasing function of time. The production cost is greater than the remanufacturing cost. The product considered in this paper is perishable product so; items deteriorate while they are in storage. To reduce the rate of product deterioration, producer is allowed to invest on the preservation technology. In this paper, the multi item inventory model developed coordinates joint production and remanufacturing options by producing new items of a product as well as by remanufacturing collected used/returned/defective items to quality standards that are "as good-as those of new products" with a single remanufacturing and a single production cycles per time interval and then, items are subject to inspection process, where item that conforms to certain quality standards is delivered to a primary market and the rest (deteriorated items from the three stocks) are shipped to a secondary market. To make this study more realistic and present environment suitable the effect of inflation and time value is also considered. We then provide a simple algorithm to find the optimal replenishment schedule and total cost for the proposed model. Finally, a couple of numerical examples are discussed to illustrate the algorithm and results are also presented graphically. The remainder of this paper is organized as follows. Section 2 provides assumptions, notations, description of the production and remanufacturing inventory systems. Section 3 is for mathematical modelling. Section 4 provides solution procedures for the resulting model. Section 5 is for illustrative examples and discusses results, while convexity of the total cost function and concluding remarks are provided in Section 6.

\section{Assumptions and notations}

In this paper, we shall establish a multi-item reverse logistics model under the following assumptions: 
We consider multi items, $i=1,2, \ldots, j$ and we will use throughout the paper the subscript " $\mathrm{r}$ " to indicate the quantity corresponds to the remanufactured stock, the subscript " $\mathrm{m}$ " to indicate the quantity corresponds to the manufactured stock, and the subscript " $R$ " to indicate the quantity corresponds to the returned stock. So, for example, $I_{i r}(t)$ represents the inventory level for ith item at time $t_{i}$ in the remanufacturing stock.

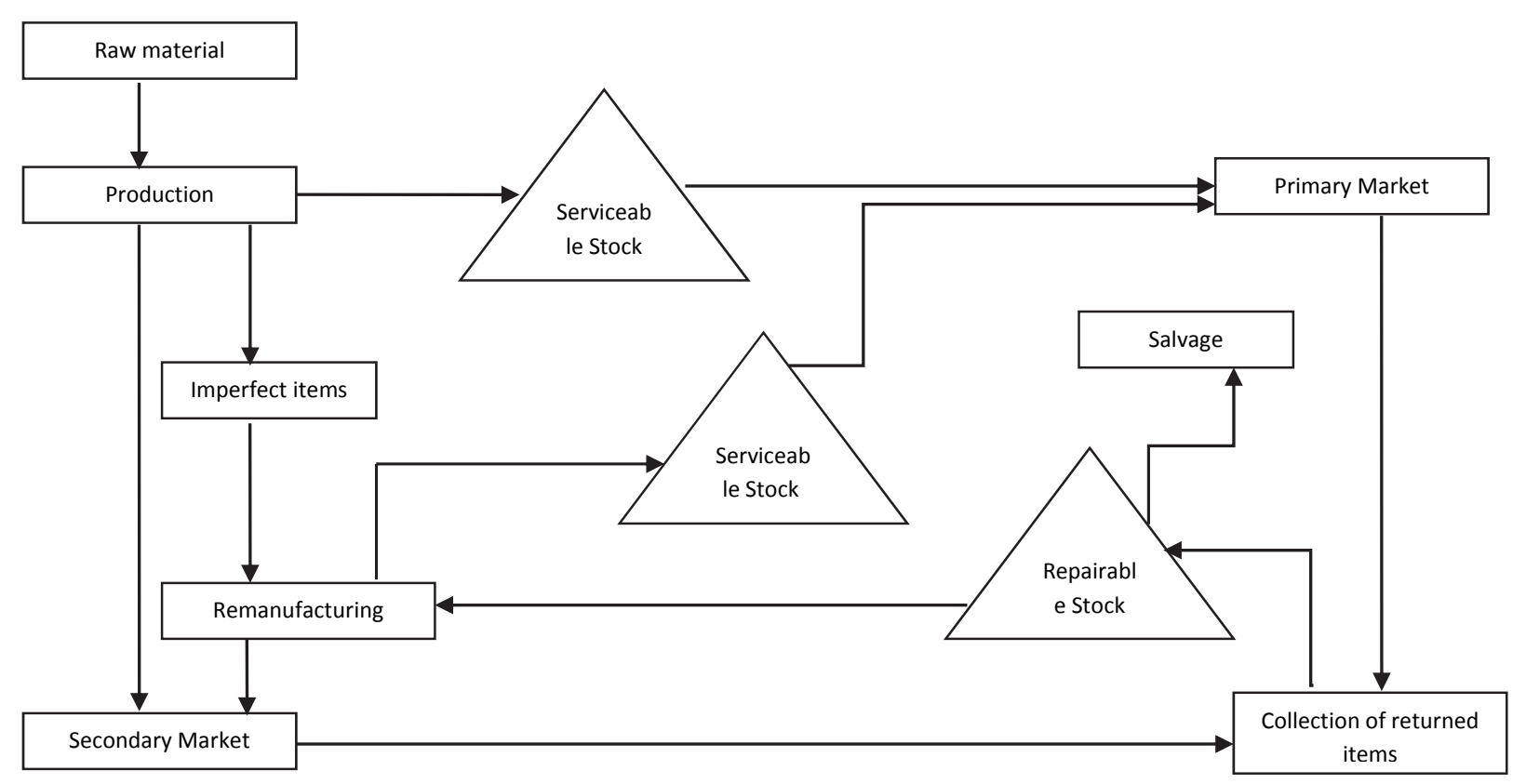

Fig. 1. General framework of products flow in one cycle

\subsection{Assumptions}

In developing the mathematical model, the following assumptions are made:

1. The model is developed for multiple items.

2. Production rate of new items and remanufacturing rate of collected returned items are linear function of time.

3. Demand rates for production and remanufacturing cycle are increasing function of time.

4. The demand rate is satisfied from a collection of newly produced and remanufactured items.

5. Used items are collected from primary and secondary market at a rate $\delta_{m} D_{m}$ and $\delta_{r} D_{r}$ respectively.

6. Preservation technology is considered for production and remanufacturing cycle.

7. The deterioration rate of returned items is time dependent.

8. The buyback products are collected from the market, where Supplier remanufactures only those used material whose quality level is higher than the level required by the market and the rest of the products are salvaged.

9. Holding cost is an exponentially increasing function of time.

10. The replenishment rate is infinite and lead time is zero.

11. Shortages are not allowed.

12. There is no repair or replacement of deteriorated items

13. Model is developed under inflationary and learning environment.

\subsection{Notations}

The following notation is used throughout this paper: 
$T_{i}$ : replenishment cycle

$\mathrm{k}$ : inflation rate

$\theta$ : Original deterioration rate of on-hand-stock, $\theta>0$

$\xi$ : Preservation technology(PT) cost for reducing deterioration rate in order to preserve the products, $\xi$ $\geq 0$.

$D_{i m}(t)$ : Demand rate for $\mathrm{i}^{\text {th }}$ item for primary market, $D_{i m}(t)=\alpha_{i} e^{\beta t}$, increases with time, where $\alpha_{i}>0$ is the initial demand rate for $\mathrm{i}^{\text {th }}$ item and $0 \leq \beta \leq 1$.

$D_{i r}(t)$ : Demand rate for $\mathrm{i}^{\text {th }}$ item for secondary market, $D_{i r}(t)=A_{i} e^{\gamma t}$, increases with time, where $A_{i}>0$ is the initial demand rate for $\mathrm{i}^{\text {th }}$ item and $0 \leq \gamma \leq 1$.

\section{Notations related to production cycle}

$P_{i m}(t)$ : Production rate for $\mathrm{i}^{\text {th }}$ item given by, $P_{m}(t)=a_{i}+b_{i} t=(1-\rho)\left(a_{i}^{\prime}+b_{i}^{\prime} t\right)$, where $b_{i}, b_{i}^{\prime}>0$, $a_{i}=a_{i}^{\prime}(1-\rho), b_{i}=(1-\rho) b_{i}^{\prime}$ and $P_{i m}(t)>D_{i m}(t)$.

$\pi(\xi)$ : Reduced deterioration rate, a function of $\xi$

$\theta_{m}:$ Resultant deterioration rate, $\theta_{m}=\theta-\pi(\xi)$

$S_{i m}+\frac{S_{m L}}{n^{v}}$ : Setup cost per cycle for $\mathrm{i}^{\text {th }}$ item with learning effect

$H_{\text {im }} e^{\Delta t}$ : Unit holding cost per unit per unit time for $\mathrm{i}^{\text {th }}$ item, where $0 \leq \Delta \leq 1$

$A_{i m}+\frac{A_{m L}}{n^{v}}$ : Unit production cost for $\mathrm{i}^{\text {th }}$ item, which includes cost components such as labour, energy, and machinery with learning effect

$C_{i m}+\frac{C_{m L}}{n^{v}}:$ Unit cost for $\mathrm{i}^{\text {th }}$ item, which includes materials cost with learning effect

\section{Notations related to remanufacturing cycle}

$P_{\text {ir }}(t)$ : Production rate for $\mathrm{i}^{\text {th }}$ item given by, $P_{\text {ir }}(t)=c_{i}+d_{i} t$, where $d_{i}>0$ and $P_{\text {ir }}(t)>D_{\text {ir }}(t)$.

$\omega(\xi)$ Reduced deterioration rate, a function of $\xi$

$\theta_{r}$ : Resultant deterioration rate, $\theta_{r}=\theta-\omega(\xi)$

$S_{i r}+\frac{S_{r L}}{n^{v}}$ : Setup cost per cycle for $\mathrm{i}^{\text {th }}$ item with learning effect

$H_{\text {ir }} e^{\Delta t}$ : Unit holding cost per unit per unit time for $\mathrm{i}^{\text {th }}$ item, where $0 \leq \Delta \leq 1$

$A_{i r}+\frac{A_{r L}}{n^{v}}$ : Unit remanufacturing cost for $\mathrm{i}^{\text {th }}$ item, which includes cost components such as labour, energy, and machinery with learning effect

\section{Notations related to returned cycle}

$R_{i}:$ The returned rate for $\mathrm{i}^{\text {th }}$ item, $R_{i}=\eta_{m} \delta_{m} D_{i m}+\eta_{r} \delta_{r} D_{i r}$

$\delta_{m}$ : Scaling parameter, returned rate formulation (from primary market)

$\delta_{r}$ : Scaling parameter, returned rate formulation (from secondary market)

$\eta_{m}$ : Scaling parameter, salvage formulation (from primary market)

$\eta_{r}$ : Scaling parameter, salvage formulation (from secondary market)

$\theta t$ : Time dependent deterioration rate

$S_{i}+\frac{S_{L}}{n^{v}}:$ Salvage for $\mathrm{i}^{\text {th }}$ item with learning effect

$S_{i R}+\frac{S_{R L}}{n^{v}}$ : Order cost per cycle for $\mathrm{i}^{\text {th }}$ item with learning effect

$H_{i R} e^{\Delta t}$ : Unit holding cost per unit per unit time for $\mathrm{i}^{\text {th }}$ item, where $0 \leq \Delta \leq 1$

$C_{i R}+\frac{C_{R L}}{n^{v}}$ : Unit cost for $\mathrm{i}^{\text {th }}$ item, which includes purchase cost with learning effect

A realization of the inventory level in the system is depicted in Figure 2. 


\section{Formulation of the model}

We have formulated a multi items production remanufacturing system. For each remanufacturing cycle, $I_{i r}(t)$ denotes the inventory level at time $t_{i}$. The system starts operating at time $t=t_{i 0}$ (initially $t_{i 0}=$ 0 ) by which the remanufacturing process starts and the inventory level increases until time $t=t_{i 1}$ and inventory level reaches its maximum, and the remanufacturing process stopped. Then the inventory level declines continuously due to combined effect of demand and deterioration and it becomes zero at time $t=t_{i 2}$ (the end of the remanufacturing cycle). The process is repeated. Hence, the inventory level during the remanufacturing cycle can be represented by the following differential equations:

$$
I_{i r}^{\prime}(t)= \begin{cases}P_{i r}(t)-D_{i r}(t)-\theta_{r} I_{i r}(t), & t_{i 0} \leq t \leq t_{i 1}, \\ -D_{i r}(t)-\theta_{r} I_{i r}(t), & t_{i 1} \leq t \leq t_{i 2}\end{cases}
$$

With the initial condition $I_{i r}(t)=0$ at $t_{i 0}\left(t_{i 0}=0\right)$ and ending condition $I_{i r}(t)=0$ at $t_{i 2}$.

$$
I_{i r}(t)= \begin{cases}{\left[\frac{c_{i}}{\theta_{r}}\left(1-e^{-\theta_{r} t}\right)-\frac{d_{i}}{\theta_{r}^{2}}\left(1-e^{-\theta_{r} t}\right)+\frac{d_{i}}{\theta_{r}} t-\frac{A_{i}}{\left(\theta_{r}+\gamma\right)}\left(e^{\gamma t}-e^{-\theta_{r} t}\right)\right],} & t_{i 0} \leq t \leq t_{i 1}, \\ \frac{A_{i}}{\left(\theta_{r}+\gamma\right)}\left[e^{\left(\theta_{r}+\gamma\right) t_{i 2}} e^{-\theta_{r} t}-e^{\gamma t}\right], & t_{i 1} \leq t \leq t_{i 2},\end{cases}
$$

Similarly, for each production cycle, $I_{i m}(t)$ denotes the inventory level at time $t_{i}$. The system starts operating at time $t=t_{i 2}$ by which the production process starts and the inventory level increases until time $t=t_{i 3}$ where the inventory level reaches its maximum, and the production process stopped. Then the inventory level declines continuously due to combined effect of demand and deterioration and it becomes zero by time $t=T_{i}$ (the end of the production cycle). The process is repeated. Hence, the inventory level during the production cycle can be represented by the following differential equations:

$$
I_{i m}^{\prime}(t)= \begin{cases}P_{i m}(t)-D_{i m}(t)-\theta_{m} I_{i m}(t), & t_{i 2} \leq t \leq t_{i 3}, \\ I_{i m}^{\prime}(t)=-D_{i m}(t)-\theta_{m} I_{i m}(t), & t_{i 3} \leq t \leq T_{i},\end{cases}
$$

With the initial condition $I_{i m}(t)=0$ at $t_{i 2}$ and ending condition $I_{i m}(t)=0$ at $T_{i}$.

$$
I_{i m}(t)= \begin{cases}{\left[\frac{a_{i}}{\theta_{m}}\left(1-e^{-\theta_{m}\left(t_{i 2}-t\right)}\right)+\frac{b_{i}}{\theta_{m}}\left(t-t_{i 2} e^{-\theta_{m}\left(t_{i 2}-t\right)}\right)-\frac{b_{i}}{\theta_{m}^{2}}\left(1-e^{-\theta_{m}\left(t_{i 2}-t\right)}\right)\right.} & t_{i 2} \leq t \leq t_{i 3}, \\ \left.-\frac{\alpha_{i}}{\left(\theta_{m}+\beta\right)}\left\{e^{\beta t}-e^{\left(\theta_{m}+\beta\right) t_{i 2}} e^{-\theta_{m} t}\right\}\right], & t_{i 3} \leq t \leq T_{i}, \\ \frac{\alpha_{i}}{\left(\theta_{m}+\beta\right)}\left[e^{\left(\theta_{m}+\beta\right) T_{i}} e^{-\theta_{m} t}-e^{\beta t}\right], & \end{cases}
$$

Also, for each returned cycle, $I_{i R}(t)$ denotes the inventory level at time $t_{i}$. As the remanufacturing system starts operating at time $t=t_{i 0}$, the inventory level decreases due to the combined effect of demand and deterioration until time $t=t_{i 1}$ by which the remanufacturing process ceased and the inventory level becomes zero. Then the inventory level starts to go up until time $t=T_{i}$ where the inventory level reaches its maximum. The process is repeated. Hence, the inventory level during the returned cycle can be represented by the following differential equations:

$$
I_{i R}^{\prime}(t)= \begin{cases}-P_{i r}(t)+R_{i}(t)-\theta t I_{i R}(t), & t_{i 0} \leq t \leq t_{i 1}, \\ R_{i}(t)-\theta t I_{i R}(t), & t_{i 1} \leq t \leq T_{i},\end{cases}
$$


where $\phi_{i r}=\eta_{r} \delta_{r} A_{i}$ and $\phi_{i m}=\eta_{m} \delta_{m} \alpha_{i}$, With the ending condition $I_{i R}(t)=0$ at $t_{i 1}$ and initial condition $I_{i R}(t)=0$ at $t_{i 1}$.

$$
I_{i R}(t)= \begin{cases}\left.\left\{\left(\frac{\left.\phi_{i r}+\phi_{i m}-c_{i}\right)\left\{\left(t-t_{i 1}\right)+\frac{\theta}{2}\left(\frac{t^{3}-t_{i 1}^{3}}{3}\right)\right\}+\left(\phi_{i r} \gamma+\phi_{i m} \beta-d_{i}\right)}{2}\right)+\frac{\theta}{2}\left(\frac{t^{4}-t_{i 1}^{4}}{4}\right)\right\}\right] e^{-\theta t^{2} / 2}, & t_{i 0} \leq t \leq t_{i 1}, \\ {\left[\left(\phi_{i r}+\phi_{i m}\right)\left\{\left(t-t_{i 1}\right)+\frac{\theta}{2}\left(\frac{t^{3}-t_{i 1}^{3}}{3}\right)\right\}+\left(\phi_{i r} \gamma+\phi_{i m} \beta\right)\right.} & \\ \left.\left\{\left(\frac{t^{2}-t_{i 1}^{2}}{2}\right)+\frac{\theta}{2}\left(\frac{t^{4}-t_{i 1}^{4}}{4}\right)\right\}\right] e^{-\theta t^{2} / 2}, & t_{i 1} \leq t \leq T_{i},\end{cases}
$$

The present value of setup/ ordering cost for $\mathrm{i}^{\text {th }}$ item during the replenishment cycle is follows,

Setup cost for remanufacturing cycle, $\quad O_{i r}=S_{i r}+\frac{S_{r L}}{n^{v}}$

Setup cost for production cycle, $\quad O_{i m}=S_{i m}+\frac{S_{m L}}{n^{v}}$

Ordering cost for returned cycle, $\quad O_{i R}=S_{i R}+\frac{S_{R L}}{n^{v}}$

The present value of production cost for $\mathrm{i}^{\text {th }}$ item during the replenishment cycle is as follows,

$$
\begin{aligned}
& P C_{i}=\left(A_{i m}+\frac{A_{i m L}}{n^{v}}\right) \int_{t_{i 2}}^{t_{i 3}} P_{i m}(t) \cdot e^{-k t} d t=\left(A_{i m}+\frac{A_{i m L}}{n^{v}}\right) \int_{t_{i 2}}^{t_{i 3}}\left(a_{i}+b_{i} t\right) \cdot e^{-k t} d t \\
& P C_{i}=\left(A_{i m}+\frac{A_{i m L}}{n^{v}}\right)\left[\left(a_{i}+\frac{b_{i}}{k}\right)\left(\frac{e^{-k t_{i 2}}-e^{-k t_{i 3}}}{k}\right)+\frac{b_{i}}{k}\left(t_{i 2} e^{-k t_{i 2}}-t_{i 3} e^{-k t_{i 3}}\right)\right]
\end{aligned}
$$

The present value of remanufacturing cost for $\mathrm{i}^{\text {th }}$ item during the replenishment cycle is as follows,

$$
\begin{aligned}
& R C_{i}=\left(A_{i r}+\frac{A_{i r L}}{n^{v}}\right)_{0}^{t_{i 1}} P_{i r}(t) \cdot e^{-k t} d t=\left(A_{i r}+\frac{A_{i r L}}{n^{v}}\right) \int_{0}^{t_{i 1}}\left(c_{i}+d_{i} t\right) \cdot e^{-k t} d t \\
& R C_{i}=\left(A_{i r}+\frac{A_{i r L}}{n^{v}}\right)\left[\left(c_{i}+\frac{d_{i}}{k}\right)\left(\frac{1-e^{-k t_{i 1}}}{k}\right)-\frac{d_{i}}{k} t_{i 1} e^{-k t_{i 1}}\right],
\end{aligned}
$$

The present value of holding cost for $\mathrm{i}^{\text {th }}$ item during the replenishment cycle is as follows, Holding cost for returned items,

$$
H C_{i R}=H_{i R}\left[\int_{0}^{t_{i 1}} I_{i R}(t) e^{-k t} d t+\int_{t_{i 1}}^{T_{i}} I_{i R}(t) e^{-k t} d t\right]
$$




$$
\begin{aligned}
& H C_{i R}=H_{i R}\left[\left[\left(\phi_{i r}+\phi_{i m}-c\right)\left\{k \frac{t_{i 1}^{3}}{6}-\frac{t_{i 1}^{2}}{2}-\frac{\theta}{2} \frac{t_{i 1}^{4}}{6}\right\}+\left(\phi_{i r} \gamma+\phi_{i m} \beta-d_{i}\right)\left\{\frac{t_{i 1}^{3}}{6}-\frac{t_{i 1}^{2}}{2}+k \frac{t_{i 1}^{4}}{8}\right\}\right]\right. \\
& +\left[\left(\phi_{i r}+\phi_{i i m}\right)\left\{\left(\frac{T_{i}^{2}}{2}-\frac{\theta}{2} \frac{T_{i}^{4}}{6}-k \frac{T_{i}^{3}}{3}\right)-\left(\frac{t_{i 1}^{2}}{2}-k \frac{t_{i 1}^{3}}{6}-\frac{\theta}{2} \frac{t_{i 1}^{4}}{6}\right)\right\}+\left(\phi_{i r} \gamma+\phi_{i m} \beta\right)\left\{\left(\frac{T_{i}^{3}}{6}-k \frac{T_{i}^{4}}{8}\right)\right.\right. \\
& \left.-\left(\frac{t_{i 1}^{3}}{6}-k \frac{t_{i 1}^{4}}{8}\right)\right\}-\left\{\left(\phi_{i r}+\phi_{i m}\right)\left(t_{i 1}+\frac{\theta}{2} \frac{t_{i 1}^{3}}{3}\right)+\left(\phi_{i r} \gamma+\phi_{i m} \beta\right)\left(\frac{t_{i 1}^{2}}{2}+\frac{\theta}{2} \frac{t_{i 1}^{4}}{4}\right)\right\}\left\{\left(T_{i}-k \frac{T_{i}^{2}}{2}-\theta \frac{T_{i}^{3}}{6}\right)\right. \\
& \left.\left.-\left(t_{i 1}-k \frac{t_{i 1}^{2}}{2}-\theta \frac{t_{i 1}^{3}}{6}\right)\right]\right]
\end{aligned}
$$

Holding cost for remanufactured items,

$$
\begin{aligned}
H C_{i r}= & H_{i r}\left[\int_{0}^{t_{11}} I_{i r}(t) e^{\Delta t} e^{-k t} d t+\int_{t_{i 1}}^{t_{i 2}} I_{i r}(t) e^{\Delta t} e^{-k t} d t\right] \\
H C_{i r} & =H_{i r}\left[\left[\frac{1}{\theta_{r}}\left(c_{i}-\frac{d_{i}}{\theta_{r}}-\frac{d_{i}}{(\Delta-k)}\right)\left(\frac{e^{(\Delta-k) t_{i 1}}-1}{(\Delta-k)}\right)+\left\{\frac{A_{i}}{\left(\theta_{r}+\gamma\right)}-\frac{1}{\theta_{r}}\left(c_{i}-\frac{d_{i}}{\theta_{r}}\right)\right\}\left(\frac{e^{\left(\Delta-k-\theta_{r}\right) t_{i 1}}-1}{\left(\Delta-k-\theta_{r}\right)}\right)\right.\right. \\
& \left.+\frac{d_{i}}{\theta_{r}} t_{i 1} \frac{e^{(\Delta-k) t_{i 1}}}{(\Delta-k)}-\frac{A_{i}}{\left(\theta_{r}+\gamma\right)}\left(\frac{e^{(\gamma+\Delta-k) t_{i 1}}-1}{(\gamma+\Delta-k)}\right)\right]+\frac{A_{i}}{\left(\theta_{r}+\gamma\right)}\left[e^{\left(\theta_{r}+\gamma\right) t_{i 2}}\left(\frac{e^{\left(\Delta-k-\theta_{r}\right) t_{i 2}}-e^{\left(\Delta-k-\theta_{r}\right) t_{i 1}}}{\left(\Delta-k-\theta_{r}\right)}\right)\right. \\
& \left.\left.-\left(\frac{e^{(\gamma+\Delta-k) t_{i 2}}-e^{(\gamma+\Delta-k) t_{i 1}}}{(\gamma+\Delta-k)}\right)\right]\right]
\end{aligned}
$$

Holding cost for manufactured items,

$$
\begin{aligned}
& H C_{i m}=H_{i m}\left[\int_{t_{i 2}}^{t_{i 3}} I_{i m}(t) e^{\Delta t} e^{-k t} d t+\int_{t_{i 3}}^{T_{i i}} I_{i m}(t) e^{\Delta t} e^{-k t} d t\right] \\
& H C_{i m}=H_{i m}\left[\left[\frac{1}{\theta_{m}}\left(a_{i}-\frac{b_{i}}{\theta_{m}}\right) \int\left(\frac{e^{(\Delta-k) t_{i 3}}-e^{(\Delta-k) t_{i 2}}}{(\Delta-k)}\right)-e^{\theta_{m} t_{i 2}}\left(\frac{e^{\left(\Delta-k-\theta_{m}\right) t_{i 3}}-e^{\left(\Delta-k-\theta_{m}\right) t_{i 2}}}{\left(\Delta-k-\theta_{m}\right)}\right)\right\}+\frac{b_{i}}{\theta_{m}}\right. \\
& \left\{\left(\frac{t_{i 3} e^{(\Delta-k) t_{i 3}}-t_{i 2} e^{(\Delta-k) t_{i 2}}}{(\Delta-k)}\right)-\left(\frac{e^{(\Delta-k) t_{i 3}}-e^{(\Delta-k) t_{i 2}}}{(\Delta-k)}\right)-t_{i 2} e^{\theta_{m} t_{i 2}}\left(\frac{e^{\left(\Delta-k-\theta_{m}\right) t_{i 3}}-e^{\left(\Delta-k-\theta_{m}\right) t_{i 2}}}{\left(\Delta-k-\theta_{m}\right)}\right)\right\} \\
& \left.-\frac{\alpha_{i}}{\left(\theta_{m}+\beta\right)}\left\{\left(\frac{e^{(\beta+\Delta-k) t_{i 3}}-e^{(\beta+\Delta-k) t_{i 2}}}{(\beta+\Delta-k)}\right)-e^{\left(\theta_{m}+\beta\right) t_{i 2}}\left(\frac{e^{\left(\Delta-k-\theta_{m}\right) t_{i 3}}-e^{\left(\Delta-k-\theta_{m}\right) t_{i 2}}}{\left(\Delta-k-\theta_{m}\right)}\right)\right\}\right]+\frac{\alpha_{i}}{\left(\theta_{m}+\beta\right)} \\
& \left.\left[e^{\left(\theta_{m}+\gamma\right) T_{i}}\left(\frac{e^{\left(\Delta-k-\theta_{m}\right) T_{i}}-e^{\left(\Delta-k-\theta_{r}\right) t_{i 3}}}{\left(\Delta-k-\theta_{m}\right)}\right)-\left(\frac{e^{(\beta)+\Delta-k) t_{i 2}}-e^{(\beta)+\Delta-k) t_{i 1}}}{(\beta+\Delta-k)}\right)\right]\right]
\end{aligned}
$$



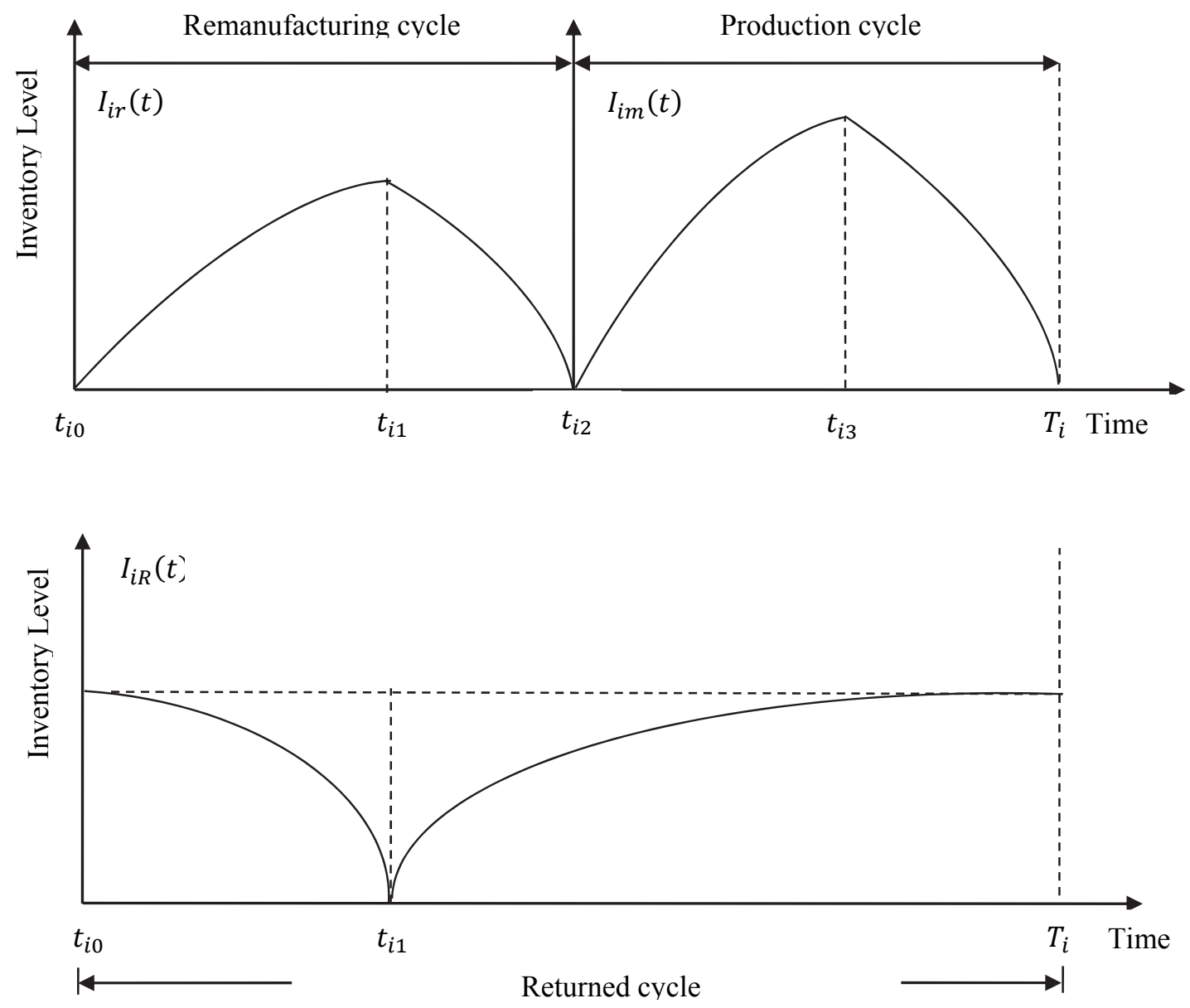

Fig. 1. Inventory variation of an EPQ model for Reverse Logistics System

The present value of salvage cost for $\mathrm{i}^{\text {th }}$ item during the replenishment cycle is as follows,

$$
S C_{i}=\left(S_{i}+\frac{S_{i L}}{n^{v}}\right)\left[\frac{\left(1-\eta_{m}\right) \delta_{m} \alpha_{i}}{(\beta-k)}\left(e^{(\beta-k) T_{i}}-1\right)+\frac{\left(1-\eta_{r}\right) \delta_{r} A_{i}}{(\gamma-k)}\left(e^{(\gamma-k) T_{i}}-1\right)\right]
$$

The present value of items cost (This cost includes the deterioration cost) during the replenishment cycle is as follows,

$$
\begin{aligned}
& I C_{i}=\left(C_{i m}+\frac{C_{i m L}}{n^{v}}\right)_{t_{i 2}}^{t_{i 3}} P_{i m}(t) \cdot e^{-k t} d t+\left(C_{i R}+\frac{C_{i R L}}{n^{v}}\right) \int_{0}^{T_{i}} R_{i}(t) \cdot e^{-k t} d t \\
& I C_{i}=\left(C_{i m}+\frac{C_{i m L}}{n^{v}}\right)\left[\left(a_{i}+\frac{b_{i}}{k}\right)\left(\frac{e^{-k t_{i 2}}-e^{-k t_{i 3}}}{k}\right)+\frac{b_{i}}{k}\left(t_{i 2} e^{-k t_{i 2}}-t_{i 3} e^{-k t_{i 3}}\right)\right] \\
& +\left(C_{i R}+\frac{C_{i R L}}{n^{v}}\right)\left[\phi_{i r}\left(\frac{e^{(\gamma-k) T_{i}}-1}{\gamma-k}\right)+\phi_{i m}\left(\frac{e^{(\beta-k) T_{i}}-1}{\beta-k}\right)\right]
\end{aligned}
$$

Thus, the total cost per unit time of the underlying multi item inventory system during the cycle $\left[0, T_{i}\right]$, which consists of the setup cost, ordering cost, production cost, remanufacturing cost, inventory 
holding cost, salvage cost and items cost (this cost includes the deterioration cost), as a function of $t_{i 1}$, $t_{i 2}, t_{i 3}$ and $T_{i}$, say $T A C_{i}\left(t_{i 1}, t_{i 2}, t_{i 3}, T_{i}\right)$ is given by

$$
\begin{aligned}
& T A C_{i}=\frac{1}{T_{i}}\left[\left(A_{i m}+\frac{A_{i m L}}{n^{v}}\right)\left[\left(a_{i}+\frac{b_{i}}{k}\right)\left(\frac{e^{-k t_{i 2}}-e^{-k t_{i 3}}}{k}\right)+\frac{b_{i}}{k}\left(t_{i 2} e^{-k t_{i 2}}-t_{i 3} e^{-k t_{i 3}}\right)\right]+\left(A_{i r}+\frac{A_{i r L}}{n^{v}}\right)\left[\left(c_{i}+\frac{d_{i}}{k}\right)\right.\right. \\
& \left.\left(\frac{1-e^{-k t_{i 1}}}{k}\right)-\frac{d_{i}}{k} t_{i 1} e^{-k t_{i 1}}\right]+H_{i R}\left[\left[\left(\phi_{i i r}+\phi_{i m}-c\right)\left\{k \frac{t_{i 1}^{3}}{6}-\frac{t_{i 1}^{2}}{2}-\frac{\theta}{2} \frac{t_{i 1}^{4}}{6}\right\}+\left(\phi_{i r} \gamma+\phi_{i m} \beta-d_{i}\right)\left\{\frac{t_{i 1}^{3}}{6}-\frac{t_{i 1}^{2}}{2}+k \frac{t_{i 1}^{4}}{8}\right\}\right]\right. \\
& +\left[\left(\phi_{i r}+\phi_{i i m}\right)\left\{\left(\frac{T_{i}^{2}}{2}-\frac{\theta}{2} \frac{T_{i}^{4}}{6}-k \frac{T_{i}^{3}}{3}\right)-\left(\frac{t_{i 1}^{2}}{2}-k \frac{t_{i 1}^{3}}{6}-\frac{\theta}{2} \frac{t_{i 1}^{4}}{6}\right)\right\}+\left(\phi_{i r} \gamma+\phi_{i m} \beta\right)\left\{\left(\frac{T_{i}^{3}}{6}-k \frac{T_{i}^{4}}{8}\right)-\left(\frac{t_{i 1}^{3}}{6}-k \frac{t_{i 1}^{4}}{8}\right)\right\}\right. \\
& \left.\left.-\left\{\left(\phi_{i r}+\phi_{i m}\right)\left(t_{i 1}+\frac{\theta}{2} \frac{t_{i 1}^{3}}{3}\right)+\left(\phi_{i r} \gamma+\phi_{i m} \beta\right)\left(\frac{t_{i 1}^{2}}{2}+\frac{\theta}{2} \frac{t_{i 1}^{4}}{4}\right)\right\}\left\{\left(T_{i}-k \frac{T_{i}^{2}}{2}-\theta \frac{T_{i}^{3}}{6}\right)-\left(t_{i 1}-k \frac{t_{i 1}^{2}}{2}-\theta \frac{t_{i 1}^{3}}{6}\right)\right\}\right]\right]+H_{i r} \\
& {\left[\left[\frac{1}{\theta_{r}}\left(c_{i}-\frac{d_{i}}{\theta_{r}}-\frac{d_{i}}{(\Delta-k)}\right)\left(\frac{e^{(\Delta-k) t_{i 1}}-1}{(\Delta-k)}\right)+\left\{\frac{A_{i}}{\left(\theta_{r}+\gamma\right)}-\frac{1}{\theta_{r}}\left(c_{i}-\frac{d_{i}}{\theta_{r}}\right)\right\}\left(\frac{e^{\left(\Delta-k-\theta_{r}\right)_{i 1}}-1}{\left(\Delta-k-\theta_{r}\right)}\right)+\frac{d_{i}}{\theta_{r}} t_{i 1} \frac{e^{(\Delta-k) t_{i 1}}}{(\Delta-k)}-\right.\right.} \\
& \left.\left.\frac{A_{i}}{\left(\theta_{r}+\gamma\right)}\left(\frac{e^{(\gamma+\Delta-k) t_{i 1}}-1}{(\gamma+\Delta-k)}\right)\right]+\frac{A_{i}}{\left(\theta_{r}+\gamma\right)}\left[e^{\left(\theta_{r}+\gamma\right)_{i 2}}\left(\frac{e^{\left(\Delta-k-\theta_{r}\right) t_{i 2}}-e^{\left(\Delta-k-\theta_{r}\right) t_{i 1}}}{\left(\Delta-k-\theta_{r}\right)}\right)-\left(\frac{e^{(\gamma+\Delta-k) t_{i 2}}-e^{(\gamma+\Delta-k) t_{i 1}}}{(\gamma+\Delta-k)}\right)\right]\right]+H_{i m} \\
& {\left[\left[\frac{1}{\theta_{m}}\left(a_{i}-\frac{b_{i}}{\theta_{m}}\right)\left\{\left(\frac{e^{(\Delta-k) t_{i 3}}-e^{(\Delta-k) t_{i 2}}}{(\Delta-k)}\right)-e^{\theta_{m} t_{i 2}}\left(\frac{e^{\left(\Delta-k-\theta_{m}\right) t_{i 3}}-e^{\left(\Delta-k-\theta_{m}\right) t_{i 2}}}{\left(\Delta-k-\theta_{m}\right)}\right)\right\}+\frac{b_{i}}{\theta_{m}}\left\{\left(\frac{t_{i 3} e^{(\Delta-k) t_{i 3}}-t_{i 2} e^{(\Delta-k) t_{i 2}}}{(\Delta-k)}\right)\right.\right.\right.} \\
& \left.-\left(\frac{e^{(\Delta-k) t_{i 3}}-e^{(\Delta-k) t_{i 2}}}{(\Delta-k)}\right)-t_{i 2} e^{\theta_{m} t_{22}}\left(\frac{e^{\left(\Delta-k-\theta_{m}\right) t_{i 3}}-e^{\left(\Delta-k-\theta_{m}\right) t_{i 2}}}{\left(\Delta-k-\theta_{m}\right)}\right)\right\}-\frac{\alpha_{i}}{\left(\theta_{m}+\beta\right)}\left\{\left(\frac{e^{(\beta+\Delta-k) t_{i 3}}-e^{(\beta+\Delta-k) t_{i 2}}}{(\beta+\Delta-k)}\right)-e^{\left(\theta_{m}+\beta\right) t_{i 2}}\right. \\
& \left.\left.\left.\left(\frac{e^{\left(\Delta-k-\theta_{m}\right) t_{i 3}}-e^{\left(\Delta-k-\theta_{m}\right) t_{i 2}}}{\left(\Delta-k-\theta_{m}\right)}\right)\right\}\right]+\frac{\alpha_{i}}{\left(\theta_{m}+\beta\right)}\left[e^{\left(\theta_{m}+\gamma\right) T_{i}}\left(\frac{e^{\left(\Delta-k-\theta_{m}\right) T_{i}}-e^{\left(\Delta-k-\theta_{r}\right) t_{i 3}}}{\left(\Delta-k-\theta_{m}\right)}\right)-\left(\frac{e^{(\beta)+\Delta-k) t_{i 2}}-e^{(\beta)+\Delta-k) t_{i 1}}}{(\beta+\Delta-k)}\right)\right]\right] \\
& -\left(S_{i}+\frac{S_{i L}}{n^{v}}\right)\left[\frac{\left(1-\eta_{m}\right) \delta_{m} \alpha_{i}}{(\beta-k)}\left(e^{(\beta-k) T_{i}}-1\right)+\frac{\left(1-\eta_{r}\right) \delta_{r} A_{i}}{(\gamma-k)}\left(e^{(\gamma-k) T_{i}}-1\right)\right]+\left(C_{i m}+\frac{C_{i m L}}{n^{v}}\right)\left[\left(a_{i}+\frac{b_{i}}{k}\right)\left(\frac{e^{-k t_{i 2}}-e^{-k t_{i 3}}}{k}\right)\right. \\
& \left.+\frac{b_{i}}{k}\left(t_{i 2} e^{-k t_{i 2}}-t_{i 3} e^{-k t_{i 3}}\right)\right]+\left(C_{i R}+\frac{C_{i R L}}{n^{\nu}}\right)\left[\phi_{i r}\left(\frac{e^{(\gamma-k) T_{i}}-1}{\gamma-k}\right)+\phi_{i m}\left(\frac{e^{(\beta-k) T_{i}}-1}{\beta-k}\right)\right]+\left(S_{i R}+\frac{S_{i R L}}{n^{\nu}}\right)+\left(S_{i r}+\frac{S_{i r L}}{n^{\nu}}\right)+ \\
& \left.\left(S_{i m}+\frac{S_{i m L}}{n^{v}}\right)\right] \\
& T A C=\sum_{i=1}^{j} T A C_{i}
\end{aligned}
$$

Here we have a cost function in terms of $t_{i 1}, t_{i 2}, t_{i 3}$ and $T_{i}$. Our goal is to find $t_{i 1}, t_{i 2}, t_{i 3}$ and $T_{i}$ that minimize $T A C_{i}\left(t_{i 1}, t_{i 2}, t_{i 3}, T_{i}\right)$, the variables $t_{i 1}, t_{i 2}, t_{i 3}$ and $T_{i}$ are related to each other through the following relations:

$$
\begin{aligned}
& 0<t_{i 1}<t_{i 2}<t_{i 3}<T_{i} \\
& {\left[\frac{c_{i}}{\theta_{r}}\left(1-e^{-\theta_{r} t_{i 1}}\right)-\frac{d_{i}}{\theta_{r}^{2}}\left(1-e^{-\theta_{r} t_{i 1}}\right)+\frac{d_{i}}{\theta_{r}} t_{i 1}-\frac{A_{i}}{\left(\theta_{r}+\gamma\right)}\left(e^{\gamma t_{i 1}}-e^{-\theta_{r} t_{i 1}}\right)\right]=\frac{A_{i}}{\left(\theta_{r}+\gamma\right)}\left[e^{\left(\theta_{r}+\gamma\right) t_{i 2}} e^{-\theta_{r} t_{i 1}}-e^{\gamma t_{i 1}}\right],}
\end{aligned}
$$




$$
\begin{aligned}
& {\left[\frac{a_{i}}{\theta_{m}}\left(1-e^{-\theta_{m}\left(t_{i 2}-t_{i 3}\right)}\right)+\frac{b_{i}}{\theta_{m}}\left(t_{i 3}-t_{i 2} e^{-\theta_{m}\left(t_{i 2}-t_{i 3}\right)}\right)-\frac{b_{i}}{\theta_{m}^{2}}\left(1-e^{-\theta_{m}\left(t_{i 2}-t_{i 3}\right)}\right)-\frac{\alpha_{i}}{\left(\theta_{m}+\beta\right)}\right.} \\
& \left.\left\{e^{\beta t_{i 3}}-e^{\left(\theta_{m}+\beta\right) t_{i 2}} e^{-\theta_{m} t_{i 3}}\right\}\right]=\frac{\alpha_{i}}{\left(\theta_{m}+\beta\right)}\left[e^{\left(\theta_{m}+\beta\right) T_{i}} e^{-\theta_{m} t_{i 3}}-e^{\beta t_{i 3}}\right], \\
& {\left[\left(c_{i}-\phi_{i r}+\phi_{i m}\right)\left\{\left(t_{i 1}\right)+\frac{\theta}{2}\left(\frac{t_{i 1}^{3}}{3}\right)\right\}-\left(\phi_{i r} \gamma+\phi_{i m} \beta-d_{i}\right)\left\{\left(\frac{t_{i 1}^{2}}{2}\right)+\frac{\theta}{2}\left(\frac{t_{i 1}^{4}}{4}\right)\right\}\right]} \\
& =\left[\left(\phi_{i r}+\phi_{i m}\right)\left\{\left(T_{i}-t_{i 1}\right)+\frac{\theta}{2}\left(\frac{T_{i}^{3}-t_{i 1}^{3}}{3}\right)\right\}+\left(\phi_{i r} \gamma+\phi_{i m} \beta\right)\left\{\left(\frac{T_{i}^{2}-t_{i 1}^{2}}{2}\right)+\frac{\theta}{2}\left(\frac{T_{i}^{4}-t_{i 1}^{4}}{4}\right)\right\}\right] e^{-\theta T_{i}^{2} / 2}
\end{aligned}
$$

Eq. (18) represents the natural monotonicity constraints, since otherwise the given problem, would have no meaning. Eq. (19) and (20) ensure the fact that the inventory levels for the remanufacturing and the production systems must have equal values for $t=t_{i 1}$ and for $t=t_{i 3}$, respectively. Eq. (21) ensures the fact that the inventory level for the collected returned items must have an equal value for $t=t_{i 0}$ and for $t=T_{i}$.

\section{Optimal solution procedure}

From Eq. (19) we note that $t_{i 2}$ can be determined as a function of $t_{i 1}$ as follows,

$$
t_{i 2}=f\left(t_{i 1}\right)
$$

From Eq. (21) we find that $T_{i}$ can be determined as a function of $t_{i 1}$ as follows,

$$
T_{i}=f\left(t_{i 1}\right)
$$

From Eq. (20) and Eq. (22) we find that $t_{i 3}$ can be determined as a function of $t_{i 2}$ and $T_{i}$, hence of $t_{i 1}$ as follows,

$$
t_{i 3}=f\left(t_{i 1}\right)
$$

Thus, if we substitute Eqs. (22-24) in Eq. (17) then the total average cost function will be the function of $t_{i 1}$. To minimize the total average cost, we will find optimal $t_{i 1}$ with the help of following solution procedure. Equations are highly non-linear so we use computer software MATHEMATICA 8.0, to solve these equations. In addition, the following solution procedure is used to derive the optimal $t_{i 1}$ value; this will help managers to determine optimal replenishment policy for the minimum total average cost.

Step 1: Put the values of other parameters.

Step 2: Differentiate the total cost Eq. (17) with respect to $t_{i 1}$ and equate to 0 . To find the $t_{i 1}^{*}$ (optimal value of $t_{i 1}$ ) i.e.

And check for the following condition

$$
\frac{d T A C_{i}\left(t_{i 1}\right)}{d t_{i 1}}=0
$$

$$
\frac{d^{2} T A C_{i}\left(t_{i 1}\right)}{d t^{2}{ }_{i 1}}>0
$$

If $t_{i 1}$ satisfies the condition, Substitute $t_{i 1}^{*}$ into (22), (23), (24) to obtain $t_{i 2}^{*}, t_{i 3}^{*}, T_{i 1}^{*}$. 
Step 3: Substitute $t_{i 1}^{*}, t_{i 2}^{*}, t_{i 3}^{*}$ and $T_{i 1}^{*}$ into (17) to obtain $T A C_{i}\left(t_{i 1}^{*}\right)$.

Step 4: Now repeat the solution procedure for the rest of the items.

\section{Numerical example and sensitivity analysis}

\subsection{Numerical Example}

The model has been explored numerically as well. There are following data which are used to explain the model, based on the studies in proper unit. We have studied this model for two items. The following numerical study has been used to find the optimal solution of the multi items production model.

$\theta=0.02, \pi(\xi)=0.005, \omega(\xi)=0.004, \beta=0.02, \gamma=0.01, \Delta=0.001, k=0.5, \eta_{m}=0.8, \delta_{m}=0.4$, $\eta_{r}=0.6, \delta_{r}=0.25$

\section{Table 1}

The results

\begin{tabular}{|c|c|c|c|c|c|c|c|c|c|c|c|c|c|c|c|c|c|}
\hline Items $i$ & $a_{i}$ & $b_{i}$ & $c_{i}$ & $d_{i}$ & $\alpha_{i}$ & $A_{i}$ & $S_{i m}$ & $S_{i r}$ & $S_{i R}$ & $H_{i m}$ & $H_{i r}$ & $H_{i R}$ & $A_{i m}$ & $A_{i r}$ & $C_{i m}$ & $C_{i R}$ & $S_{i}$ \\
\hline 1 & 250 & 10 & 500 & 20 & 30 & 80 & 400 & 300 & 200 & 5.5 & 3.5 & 1.5 & 5.1 & 2.1 & 3.1 & 0.7 & 3.1 \\
\hline 2 & 125 & 20 & 250 & 3 & 5 & 10 & 240 & 160 & 120 & 5 & 3 & 1 & 5 & 2 & 3 & 0.8 & 3 \\
\hline
\end{tabular}

When $n=1$, the total average cost for first item is 373.369 and for second item is 107.352 and net total average cost is 480.721 .

Table 2

The optimal value of $t_{i 1}, t_{i 2}, t_{i 3}, T_{i 1}$ and TAC for the first item, i.e. $i=1$

\begin{tabular}{lccccc}
\hline $\mathrm{n}$ & $\boldsymbol{t}_{\boldsymbol{i} \mathbf{1}}^{*}$ & $\boldsymbol{t}_{\boldsymbol{i} \boldsymbol{2}}^{*}$ & $\boldsymbol{t}_{\boldsymbol{i} \mathbf{3}}^{*}$ & \multicolumn{1}{c}{$\boldsymbol{T}_{\boldsymbol{i} \mathbf{1}}^{*}$} & $\boldsymbol{T A}_{\boldsymbol{A}} \boldsymbol{C}^{*}$ \\
\hline 1 & 0.0778549 & 0.487199 & 0.628212 & 1.8050 & 373.369 \\
2 & 0.0778552 & 0.487201 & 0.628214 & 1.80501 & 373.310 \\
3 & 0.0778554 & 0.487202 & 0.628216 & 1.80501 & 373.276 \\
4 & 0.0778555 & 0.487203 & 0.628217 & 1.80502 & 373.252 \\
5 & 0.0778556 & 0.487204 & 0.628217 & 1.80502 & 373.234 \\
\hline
\end{tabular}

Table 3

The optimal value of $t_{i 1}, t_{i 2}, t_{i 3}, T_{i 1}$ and TAC for the second item, i.e. $\mathrm{i}=2$

\begin{tabular}{lrrrrr}
\hline $\mathrm{n}$ & $\mathbf{t}_{\mathbf{i} 1}^{*}$ & $\mathbf{t}_{\mathbf{i} 2}^{*}$ & $\mathbf{t}_{\mathbf{i} 3}^{*}$ & $\mathbf{T}_{\mathbf{i} 1}^{*}$ & $\mathbf{T A C}_{\mathbf{i}}{ }^{*}$ \\
\hline 1 & 0.0395741 & 0.989979 & 1.06334 & 3.19222 & 107.352 \\
2 & 0.0395745 & 0.989989 & 1.06335 & 3.19225 & 107.342 \\
3 & 0.0395747 & 0.989994 & 1.06336 & 3.19227 & 107.335 \\
4 & 0.0395749 & 0.989999 & 1.06336 & 3.19228 & 107.331 \\
5 & 0.0395750 & 0.990001 & 1.06337 & 3.19229 & 107.327 \\
\hline
\end{tabular}

\subsection{Sensitivity analysis}

Sensitivity analysis is carried out when the parameters $a_{i}, c_{i}, \alpha_{i}, A_{i}$, and $\mathrm{k}$ are changed by $-20 \%$, $-10 \%, 10 \%$ and $20 \%$. Table 1 and 2 show the changes in the optimal time and the optimal total cost for variables $a_{i}, c_{i}, \mathrm{k}, \alpha_{i}$ and $A_{i}$ respectively.

Sensitivity analysis for item 1: 
Table 4

For first item, i.e. $\mathrm{i}=1$ and $\mathrm{n}=1$

\begin{tabular}{|c|c|c|c|c|c|}
\hline$a_{i}$ & $t_{i 1}^{*}$ & $t_{i 2}^{*}$ & $\boldsymbol{t}_{i 3}^{*}$ & $\boldsymbol{T}_{i 1}^{*}$ & $T A C^{*}$ \\
\hline 300 & 0.0824299 & 0.515861 & 0.64154 & 1.91122 & 323.827 \\
\hline 275 & 0.0802613 & 0.502275 & 0.635152 & 1.86088 & 321.648 \\
\hline 250 & 0.0778549 & 0.487199 & 0.628212 & 1.8050 & 373.369 \\
\hline 225 & 0.0751645 & 0.47034 & 0.620634 & 1.74253 & 319.421 \\
\hline 200 & 0.0721299 & 0.451327 & 0.612324 & 1.67206 & 252.248 \\
\hline$c_{i}$ & $t_{i 1}^{*}$ & $t_{i 2}^{*}$ & $t_{i 3}^{*}$ & $T_{i 1}^{*}$ & $T A C^{*}$ \\
\hline 600 & 0.0651439 & 0.489082 & 0.630613 & 1.81149 & 369.958 \\
\hline 550 & 0.0709419 & 0.488273 & 0.6229581 & 1.8087 & 371.413 \\
\hline 500 & 0.0778549 & 0.487199 & 0.628212 & 1.8050 & 373.369 \\
\hline 450 & 0.086232 & 0.485724 & 0.626333 & 1.79994 & 376.066 \\
\hline 400 & 0.0965802 & 0.483646 & 0.623688 & 1.79284 & 379.907 \\
\hline $\mathrm{k}$ & $t_{i 1}^{*}$ & $t_{i 2}^{*}$ & $t_{i 3}^{*}$ & $T_{i 1}^{*}$ & $T A C^{*}$ \\
\hline 0.6 & 0.0782237 & 0.489506 & 0.631222 & 1.81355 & 463.232 \\
\hline 0.55 & 0.0780283 & 0.488284 & 0.629627 & 1.80902 & 422.556 \\
\hline 0.5 & 0.0778549 & 0.487199 & 0.628212 & 1.8050 & 373.369 \\
\hline 0.45 & 0.0777091 & 0.486285 & 0.627018 & 1.80162 & 312.829 \\
\hline 0.4 & 0.0775995 & 0.485596 & 0.626119 & 1.79906 & 236.673 \\
\hline$\alpha_{i}$ & $t_{i 1}^{*}$ & $t_{i 2}^{*}$ & $t_{i 3}^{*}$ & $T_{i 1}^{*}$ & $T A C^{*}$ \\
\hline 36 & 0.0763577 & 0.477814 & 0.622566 & 1.62571 & 372.239 \\
\hline 33 & 0.0769736 & 0.481674 & 0.626332 & 1.70859 & 372.78 \\
\hline 30 & 0.0778549 & 0.487199 & 0.628212 & 1.8050 & 373.369 \\
\hline 27 & 0.0790845 & 0.4949 & 0.631754 & 1.91882 & 373.741 \\
\hline 24 & 0.0807875 & 0.505571 & 0.637689 & 2.05583 & 373.529 \\
\hline$A_{i}$ & $t_{i 1}^{*}$ & $t_{i 2}^{*}$ & $t_{i 3}^{*}$ & $T_{i 1}^{*}$ & $T A C^{*}$ \\
\hline 96 & 0.0862992 & 0.450095 & 0.595057 & 1.801 & 355.472 \\
\hline 88 & 0.0820825 & 0.466987 & 0.610158 & 1.803 & 363.391 \\
\hline 80 & 0.0778549 & 0.487199 & 0.628212 & 1.8050 & 373.369 \\
\hline 72 & 0.0736227 & 0.511866 & 0.650251 & 1.80712 & 385.95 \\
\hline 64 & 0.0693952 & 0.54275 & 0.677902 & 1.80967 & 401.883 \\
\hline
\end{tabular}

\subsection{Observations}

- One can easily observe from the results of Table 1 that as production rate parameter $\left(a_{i}\right)$ decreases there is a significant decrease in $t_{i 1}^{*}, t_{i 2}^{*}, t_{i 3}^{*}, T_{i 1}^{*}$ and the total average cost $T A C^{*}$ and if we decrease the production rate parameter $t_{i 1}^{*}, t_{i 2}^{*}, t_{i 3}^{*}, T_{i 1}^{*}$ and $T A C^{*}$ decreases. This happens because if production rate increases then the time required producing and consuming the units also increases and total average cost $T A C^{*}$ increases.

- When we increase the value of remanufacturing rate parameter $\left(c_{i}\right), t_{i 1}^{*}$ and $T A C^{*}$ decreases as well as $t_{i 2}^{*}, t_{i 3}^{*}, T_{i 1}^{*}$ increases and if we decrease the $c_{i}, t_{i 1}^{*}$ and $T A C^{*}$ increases as well as $t_{i 2}^{*}$, $t_{i 3}^{*}, T_{i 1}^{*}$ decreases.

- From the table we can observe that when the discount rate of inflation $\mathrm{k}$ is increasing, $t_{i 1}^{*}, t_{i 2}^{*}$, $t_{i 3}^{*}, T_{i 1}^{*}$ and the total average cost $T A C^{*}$ will increase and when $k$ is decreasing, $t_{i 1}^{*}, t_{i 2}^{*}, t_{i 3}^{*}, T_{i 1}^{*}$ and $T A C^{*}$ will decrease In addition, the total present value of average cost $T A C^{*}$ is more sensitive to $\mathrm{k}$ when its value is small.

- Table1. clearly shows that as the demand rate parameter $\left(\alpha_{i}\right)$ decreases the $t_{i 1}^{*}, t_{i 2}^{*}, t_{i 3}^{*}, T_{i 1}^{*}$ increases marginally, total average cost $T A C^{*}$ decreases significantly but as $\alpha_{i}$ increases, $t_{i 1}^{*}$, $t_{i 2}^{*}, t_{i 3}^{*}, T_{i 1}^{*}$ will decreases and $T A C^{*}$ also decreases.

- Moreover, it is observed form Table 1 that as demand rate parameter $\left(A_{i}\right)$ increases then $t_{i 1}^{*}$ increase while $t_{i 2}^{*}, t_{i 3}^{*}, T_{i 1}^{*}$ and the total average cost $T A C^{*}$ decreases, significantly and if we 
decrease the $A_{i}, t_{i 1}^{*}$ decreases as well as $t_{i 2}^{*}, t_{i 3}^{*}, T_{i 1}^{*}$ and $T A C^{*}$ increases This means the demand for remanufacturer should be high which results minimum total cost.

Similarly, the sensitivity analysis for item 2 can also be performed.

Table 5

For second item, i.e. $\mathrm{i}=2$ and $\mathrm{n}=1$

\begin{tabular}{|c|c|c|c|c|c|}
\hline$a_{i}$ & $t_{i 1}^{*}$ & $t_{i 2}^{*}$ & $t_{i 3}^{*}$ & $\boldsymbol{T}_{i 1}^{*}$ & $T A C$ \\
\hline 150 & 0.0402161 & 1.00605 & 1.07153 & 3.24402 & 125.56 \\
\hline 137 & 0.0398917 & 0.997929 & 1.06723 & 3.21784 & 116.797 \\
\hline 125 & 0.0395741 & 0.989979 & 1.06334 & 3.19222 & 107.352 \\
\hline 112 & 0.0392064 & 0.980775 & 1.05925 & 3.16255 & 95.2541 \\
\hline 100 & 0.0388403 & 0.971611 & 1.05565 & 3.13301 & 81.8645 \\
\hline$c_{i}$ & $t_{i 1}^{*}$ & $t_{i 2}^{*}$ & $t_{i 3}^{*}$ & $T_{i 1}^{*}$ & $T A C$ \\
\hline 300 & 0.032982 & 0.989982 & 1.06335 & 3.19233 & 107.318 \\
\hline 275 & 0.035979 & 0.989992 & 1.06336 & 3.19231 & 107.332 \\
\hline 250 & 0.039574 & 0.989979 & 1.06334 & 3.19222 & 107.352 \\
\hline 225 & 0.043967 & 0.989953 & 1.06331 & 3.19209 & 107.383 \\
\hline 200 & 0.049457 & 0.989923 & 1.06327 & 3.19196 & 107.43 \\
\hline $\mathrm{k}$ & $t_{i 1}^{*}$ & $t_{i 2}^{*}$ & $t_{i 3}^{*}$ & $T_{i 1}^{*}$ & $T A C$ \\
\hline 0.6 & 0.037463 & 0.937136 & 0.999821 & 3.02189 & 152.726 \\
\hline 0.55 & 0.038416 & 0.96099 & 1.0284 & 3.09878 & 132.708 \\
\hline 0.5 & 0.0395741 & 0.989979 & 1.06334 & 3.19222 & 107.352 \\
\hline 0.45 & 0.041004 & 1.02577 & 1.1068 & 3.30759 & 74.393 \\
\hline 0.4 & 0.042810 & 1.07098 & 1.16219 & 3.45331 & 30.157 \\
\hline$\alpha_{i}$ & $t_{i 1}^{*}$ & $t_{i 2}^{*}$ & $t_{i 3}^{*}$ & $T_{i 1}^{*}$ & $T A C$ \\
\hline 7 & 0.038966 & 0.974755 & 1.05046 & 2.55036 & 114.201 \\
\hline 6 & 0.039270 & 0.982367 & 1.0569 & 2.87129 & 110.776 \\
\hline 5 & 0.039574 & 0.989979 & 1.0633 & 3.19222 & 107.352 \\
\hline 4 & 0.040200 & 1.00565 & 1.0773 & 3.61598 & 104.174 \\
\hline 3 & 0.041313 & 1.03351 & 1.1027 & 4.19952 & 100.081 \\
\hline$A_{i}$ & $t_{i 1}^{*}$ & $t_{i 2}^{*}$ & $t_{i 3}^{*}$ & $T_{i 1}^{*}$ & $T A C$ \\
\hline 14 & 0.051394 & 0.918505 & 0.99497 & 3.47364 & 88.534 \\
\hline 12 & 0.045609 & 0.950881 & 1.02605 & 3.35452 & 96.613 \\
\hline 10 & 0.0395741 & 0.989979 & 1.06334 & 3.19222 & 107.352 \\
\hline 8 & 0.033194 & 1.03786 & 1.10861 & 2.96434 & 122.603 \\
\hline 6 & 0.026327 & 1.09742 & 1.1642 & 2.63312 & 146.77 \\
\hline
\end{tabular}

\section{Convexity and conclusion}

\subsection{Convexity}

Convexity of the reverse logistics inventory model for the whole system $T A C^{*}$ for both the items is shown in Fig. 3 and Fig. 4.

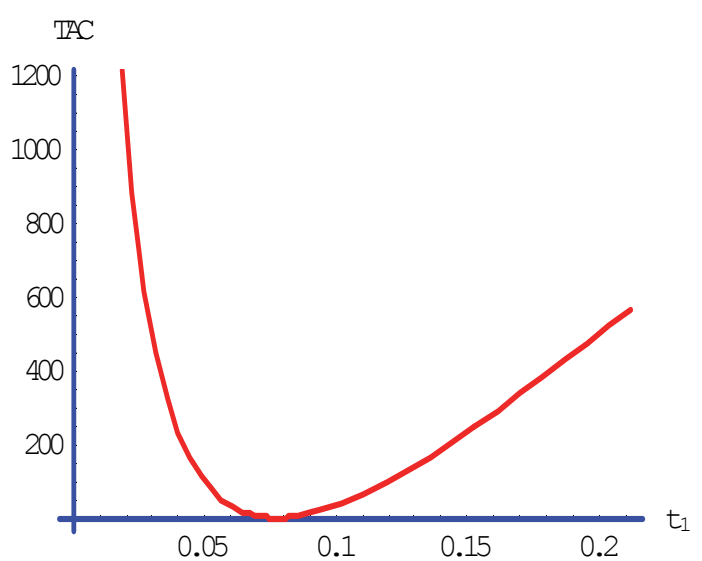

Fig. 3. Convexity of total cost $T A C^{*}$ with respect to $t_{1}$

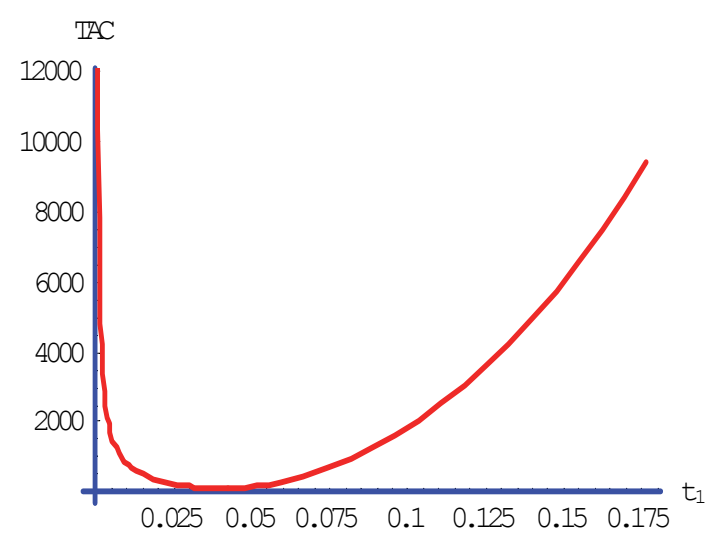

Fig. 4. Convexity of total cost $T A C^{*}$ with respect to $t_{1}$ 


\subsection{Conclusion}

This paper has presented a muti-item reverse logistics inventory model for integrated production of new items and remanufacturing of defective and returned items. The generality of our model comes from the fact that the holding cost, production, remanufacturing, demand, return, and product deterioration rates are arbitrary functions of time and production is imperfect. This study developed a deteriorating inventory model where we invest in the preservation technology (PT) cost to decrease the deterioration rate of the products and maximize the profit of the system. We have developed an analytic formulation of the problem on the framework described above and have presented an optimal solution procedure to find optimal replenishment policy. From our research results, we have verified that the effect of learning on the costs of the inventory system leads to minimize the total cost because due to the learning phenomena total average cost decreases as we increase the number of production runs. Further, a comprehensive sensitivity analysis is also carried out to show the effects of the key parameters on the optimal replenishment time and total average cost of the inventory system and the computational results show that this model can provide an efficient opportunity for managers to make proper decisions for designing logistics network among various facilities with various parameters. For future study, it is desirable to extend the proposed model for multiple production and remanufacturing batches per interval, effect of forgetting curves on production and remanufacturing rates and effect of reliability involved in the reverse logistics network design problem.

\section{References}

Badiru, A.B. (1995). Multivariate analysis of the effect of learning and forgetting on product quality. International Journal of Production Research, 33(3), 777-794.

Buzacott, J.A. (1975). Economic order quantities with inflation. Operational Research Quarterly, 26(3), 1188-1191.

Covert, R.P., \& Philip, G.C. (1973). An EOQ model with Weibull distribution deterioration. AIIE Transactions, 5(4), 323-326.

Dekker, R., Fleischmann, M., Inderfurth, K., \& van Wassenhove, L. N. (Eds.). (2013). Reverse logistics: quantitative models for closed-loop supply chains. Springer Science \& Business Media.

Dobos, I., \& Richter, K. (2003). A production/recycling model with stationary demand and return rates. Central European Journal of Operations Research, 11(1), 35-46.

Dye, CY., \& Hsieh, TP. (2012). An optimal replenishment policy for deteriorating items with effective investment in preservation technology. European Journal of Operational Research, 218(1), 106112.

Garvin, D.A. (1988). Managing Quality. Free Press, New York.

Ghare, P.M., \& Schrader, G.H. (1963). A model for an exponentially decaying inventory. Journal of Industrial Engineering, 14(5), 238-243.

Guide, V. D. R., Jayaraman, V., \& Linton, J. D. (2003). Building contingency planning for closed-loop supply chains with product recovery. Journal of operations Management, 21(3), 259-279.

Gupta, R., \& Vrat, P. (1986). Inventory model with multi-items under constraint systems for stock dependent consumption rate. Operations Research, 24(1), 41-42.

Jaber, M. Y., \& El Saadany, A. M. (2011). An economic production and remanufacturing model with learning effects. International Journal of Production Economics, 131(1), 115-127.

Jaber, M.Y., \& Bonney, M. (2003). Lot sizing with learning and forgetting in set-ups and in product quality. International Journal of Production Economics, 83(1), 95-111.

Johnson, M.R., \& Wang, M.H. (1998). Economical evaluation of disassembly operations for recycling, remanufacturing and reuse. International Journal of Production Research, 36(12), 3227-3252.

Krumwiede, D. W., \& Sheu, C. (2002). A model for reverse logistics entry by third-party providers. Omega, 30(5), 325-333. 
Liao, H. C., Tsai, C. H., \& Su, C. T. (2000). An inventory model with deteriorating items under inflation when a delay in payment is permissible.International Journal of Production Economics, 63(2), $207-$ 214.

Nahmias, S., \& Rivera, H. (1979). A deterministic model for a repairable item inventory system with a finite repair rate. International Journal of Production Research, 17(3), 215-221.

Ouyang, L. Y., Wu, K. S., \& Yang, C. T. (2006). A study on an inventory model for non-instantaneous deteriorating items with permissible delay in payments. Computers \& Industrial Engineering, 51(4), 637-651.

Ray, J., \& Chaudhuri, K. S. (1997). An EOQ model with stock-dependent demand, shortage, inflation and time discounting. International Journal of Production Economics, 53(2), 171-180.

Sarkar, B., \& Moon, I. (2011). An EPQ model with inflation in an imperfect production system. Applied Mathematics and Computation, 217(13), 6159-6167.

Schrady, D. A. (1967). A deterministic inventory model for reparable items.Naval Research Logistics Quarterly, 14(3), 391-398.

Singh, S. R., Jain, S., \& Pareek, S. (2012). A warehouse imperfect fuzzified production model with shortages under inflationary conditions. Advances in Decision Sciences, 2012, Article ID638060, 16 pages.

Singh, S., Jain, S., \& Pareek, S. (2013). An imperfect quality items with learning and inflation under two limited storage capacity. International Journal of Industrial Engineering Computations, 4(4), 479-490.

Singh, S., Prasher, L., \& Saxena, N. (2013). A centralized reverse channel structure with flexible manufacturing under the stock out situation.International Journal of Industrial Engineering Computations, 4(4), 559-570.

Singh, SR., \& Saxena, N. (2012). An Optimal Returned Policy for a Reverse Logistics Inventory Model with Backorders. Advances in Decision Sciences, Article ID 386598: 21 pages.

Wee, H., Hsu, P., \& Teng, H. (2010). Preservation technology investment for deteriorating inventory. International Journal of Production Economics, 124(2), 388-394.

Wright, T. (1936). Factors affecting the cost of airplanes. Journal of Aeronautical Science, 3(2), 122128.

Wu, K.S., Ouyang, L.Y., \& Yang, C.T. (2006). An optimal replenishment policy for non- instantaneous deteriorating items with stock-dependent demand and partial backlogging. International Journal of Production Economics, 101(2), 369-384.

Yang, P. C., Chung, S. L., Wee, H. M., Zahara, E., \& Peng, C. Y. (2013). Collaboration for a closedloop deteriorating inventory supply chain with multi-retailer and price-sensitive demand. International Journal of Production Economics, 143(2), 557-566.

Yelle, L.E. (1979). The learning curve: Historical review and comprehensive survey. Decision Sciences, 10(2), 302-328. 